\title{
Optimization Design Combined Thermal \& Deformation Evaluation for a Precision Hot-pressing Furnace
}

\author{
Yang $\mathrm{Hu}^{1,}$, Mujun $\mathrm{Li}^{1, \mathrm{~b}}$, Tianyi Shi, ${ }^{1, \mathrm{c}}$, Jian Zhou ${ }^{1, \mathrm{~d}}$, Shengzhou Huang ${ }^{1, \mathrm{e}}$ and \\ Lianguan Shen ${ }^{1, f^{*}}$ \\ ${ }^{1}$ Department of Precision Machinery and Precision Instrumentation, School of Engineering, \\ University of Science and Technology of China, Hefei, Anhui, 230027, P.R.China

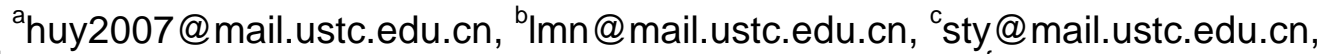 \\ dzhoujian@mail.ustc.edu.cn, ${ }^{\mathrm{e}} \mathrm{hsz2008@mail.ustc.edu.cn,} \mathrm{Igshen@ustc.edu.cn}$ \\ *corresponding author: Lianguan Shen; phone number: 86-551-63607547
}

Keywords: Optimization design, Numerical simulation, Precision Hot-pressing, Heat transfer. Abstract. With its low production cost and environment compatibility, precision compression molding of glass lenses technology has developed rapidly. In this research, a numerical optimization design for a compact and efficient precision glass molding prototype is raised, which combine both thermal and deformation analysis. As a result, the operation temperature and the deformation of sensitive parts can be controlled well. The temperature of the glass sample can reach up to over $600^{\circ} \mathrm{C}$ within 20 minutes and keep stability for a long time, meanwhile the temperature on the outside wall of furnace can be kept in a safe degree for operators. Furthermore, an effective proposal comes up to decrease the thermal deformation of the pressing rods and molds to control the manufacturing accuracy of the products.

\section{Introduction}

In recent years, with the rapid growth of market for electronic imaging products and the demand for compact high precision glass, optical components has led to increasing need for precision glass lenses. Different from conventional material removal processes including grinding, polishing and lapping, precision glass molding technology can not only avoid involving the pollutant of grinding/polishing liquids or glass debris during the manufacturing procedures, but also get rid of the follow-up finishing operations [1].

China is a manufacturing powerhouse, but related to manufacturers, these optical elements are mainly produced by imported foreign equipment, such as Moore Nanotechnology System LLC, Toshiba Machine CO, etc. Considering the new glass materials and the diversity of product demands being constantly emerging, a compact experimental glass compression molding device with flexibility in structure is self-developed.

The main objectives of this research are as follows: 1) To choose suitable heating elements for the heating source to guarantee enough power for heating glass to reach over glass transition temperature and keeping high heating efficiency. 2) To determine heat protection measures to keep stability and uniformity of the temperature at the central area and guarantee the safety of operators. 3) To design the structure to control the manufacturing accuracy of the molded glass lenses.

\section{Basic Structure Design and Material Choices}

Concept Design of the Prototype. Fig. 1 shows the concept design scheme of the precision glass molding machine which is mainly composed of a heating furnace, supports, a unit of electric actuator and a vacuum molding chamber for preventing the glass from being oxidized. 


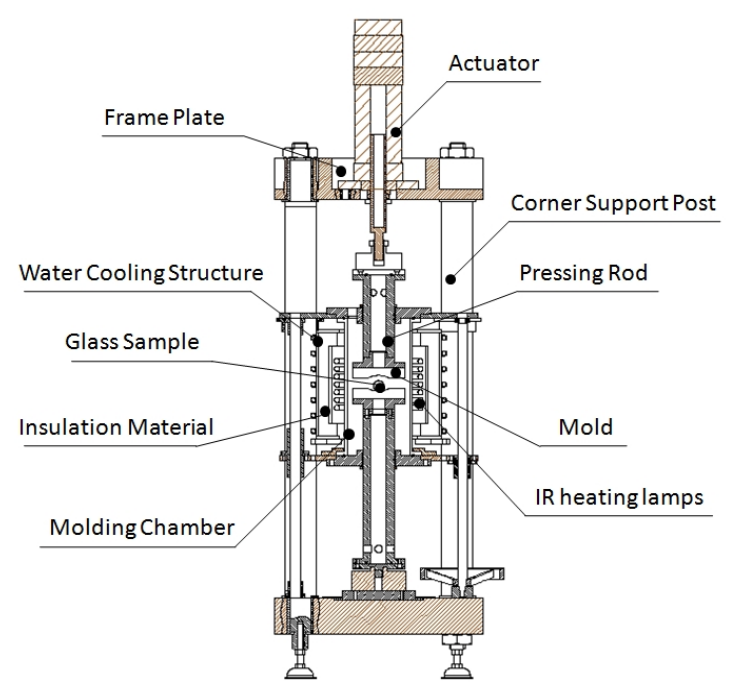

Fig. 1. Schematic illustration of the precision molding furnace

In order to simplify the vacuum system maintenance and the structure immersed in vacuum atmosphere, a quartz glass chamber is specially employed to accommodate the work pieces while heat emitters and thermal shielding structure are fixed outside the vacuum chamber.

Heat Source. In industry furnace, commonly used heating modes include resistance heating, electromagnetic induction heating and infrared (IR) heating. Resistance heating is easy to achieve, but the heat source must contact the heated body. In contrast, induction heating and IR heating are non-contact heating. Induction heating can cause rapid surface heating rate on metal, which will lead to serious non-uniformity of temperature in the heated body. In comparison, IR heating can achieve higher heating rate and better temperature homogeneity in relatively impact structure [2].

There are three kinds of IR heating: far infrared (FIR) heating, mid infrared (MIR) heating and near infrared (NIR) heating. Finally NIR heating is chosen based on these reasons: 1) the furnace chamber is made of metal, which usually has a relatively high surface absorption in NIR region; 2) NIR heating can apply and maintain higher heating rate [3].

Thermal Shielding Layers and Heat Insulation Materials. In order to enhance the heating efficiency and safety of operation, temperature in the center area of the furniture must be risen up to the glass transition temperature rapidly, which is usually demanded for over $600^{\circ} \mathrm{C}$, while temperature of the outer wall must be restricted under $60^{\circ} \mathrm{C}$ [4].

In IR heating furnace, thermal shielding layers with low emissivity between heat source and outer wall could not only prevent heat from losing through outer wall, but also effectively reflect heat back to the center. Preliminary investigation has been carried out to select a compact and effective thermal shielding and adiabatic structure. The researches show that the first thermal shielding layer is the most efficient, which brings a $150^{\circ} \mathrm{C}$ temperature enhancement in central glass area while the second layer only increases about $40^{\circ} \mathrm{C}$. Moreover, the first layer also contributes to the temperature reduction on the outer surface of furnace more effectively than the rest ones. Therefore, one thermal shielding layer combining with some insulation material can meet the constraint of compact size [5].

Several kinds of common materials such as calcium silicate product and aluminum silicate fiber are considered for the thermal insulation material. Finally the flexible silica aerogel is chosen because of its excellent performance of low thermal conductivity, low density, non-toxicity and environment friendly. Its heat conductivity is under $0.05 \mathrm{w} /(\mathrm{m} \cdot \mathrm{K})$ even when the temperature is over $800^{\circ} \mathrm{C}$ [6]. Therefore, filling it in the narrow space between the outer wall and inner wall of the furnace can maintain the large temperature gradient between them.

\section{Optimization Design of the Structure based on Temperature Field}

A 2-demension (2-D) model is built in software ANSYS Fluent to gain a fundamental understanding of the temperature distribution during heating process. Fig. 2 shows the basic simulation model of the 
furnace structure and Fig. 3 shows the temperature distribution of the furnace after heating for 20 minutes at $3 \mathrm{~kW}$.

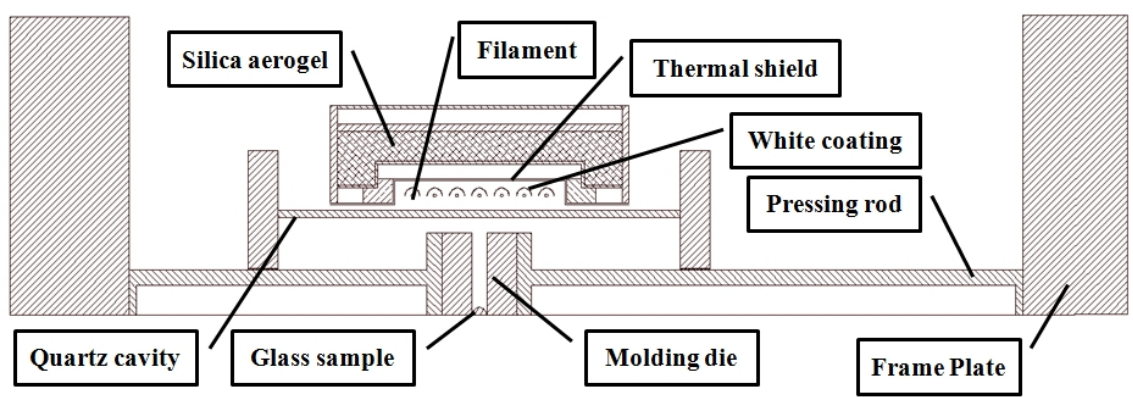

Fig. 2. 2-D model of the furnace structure
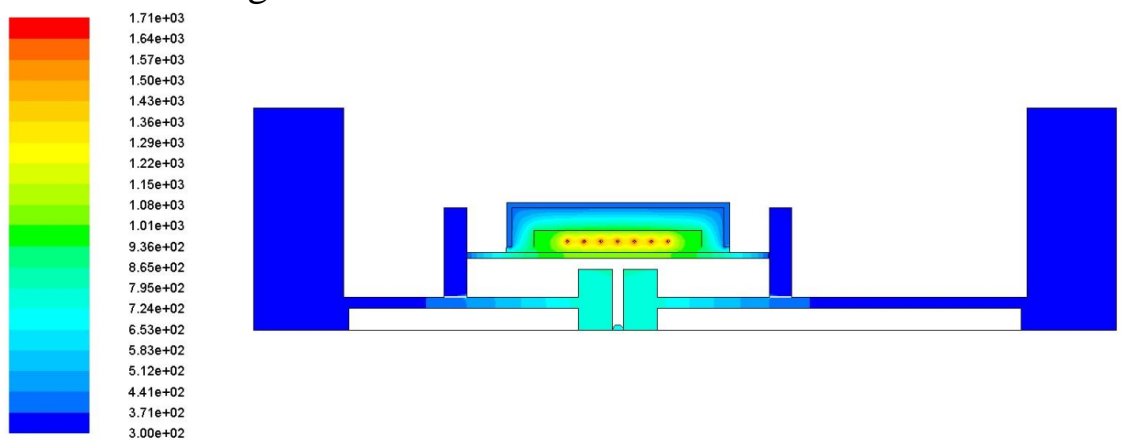

Fig. 3. Temperature distribution of the furnace after heating for 20 minutes at $3 \mathrm{~kW}$

In the furnace, two pressing rods are the main heat transfer medium. Table 2 shows the temperature in the central glass sample after heating for 20 minutes for different wall thickness of pressing rods. It can be seen that with the wall thickness increasing, temperature of the glass sample decreased significantly.

\begin{tabular}{|c|c|c|c|}
\hline Wall thickness $[\mathbf{m m}]$ & 5 & 10 & 15 \\
\hline Temperature $\left[{ }^{\circ} \mathbf{C}\right]$ & 652 & 583 & 536 \\
\hline
\end{tabular}

Table 1. Central temperature for different wall thickness of pressing rods

\section{Optimization Design of the Structure based on Thermal Deformation}

As above shown that simulations of temperature distribution of furnace can only help us optimize the structure to meet the temperature requirements. However for the precision hot-pressing furnace, deformation of different components also affects the accuracy of the molded glass sample significantly and must be considered.

As the material of mold and die is tungsten carbide (WC), the matching material of pressing rod are selected as stainless steel, silicon carbide $(\mathrm{SiC}), \mathrm{WC}$ and silicon nitride $\left(\mathrm{Si}_{3} \mathrm{~N}_{4}\right)$ to compare their comprehensive influence. Table 3 lists the thermal physical properties of these materials $[7,8]$.

\begin{tabular}{|c|c|c|}
\hline \multirow{2}{*}{ Materials } & \multicolumn{2}{|c|}{ Coefficients } \\
\cline { 2 - 3 } & Thermal conductivity $\left[\boldsymbol{W} \cdot \boldsymbol{m}^{-1} \cdot \boldsymbol{K}^{-1}\right]$ & Expansion coefficient $\left[\mathbf{1 0}^{-\mathbf{6}} \cdot \boldsymbol{K}^{-1}\right]$ \\
\hline $\mathrm{Si}_{3} \mathrm{~N}_{4}$ & 16.7 & 2.75 \\
\hline Stainless steel & 20 & 17 \\
\hline $\mathrm{SiC}$ & 64 & 5 \\
\hline $\mathrm{WC}$ & 84 & 4 \\
\hline
\end{tabular}

Table 2. Thermal physical properties of different materials

Simulations for different pressing rod materials are done under same conditions: heating for 20 minutes at $3 \mathrm{~kW}$ power. The thermal deformation of the down mold is shown in Fig.4. The calculation shows that Si3N4 is the most suitable material for pressing rod for its smallest thermal deformation of the down mold surface. 


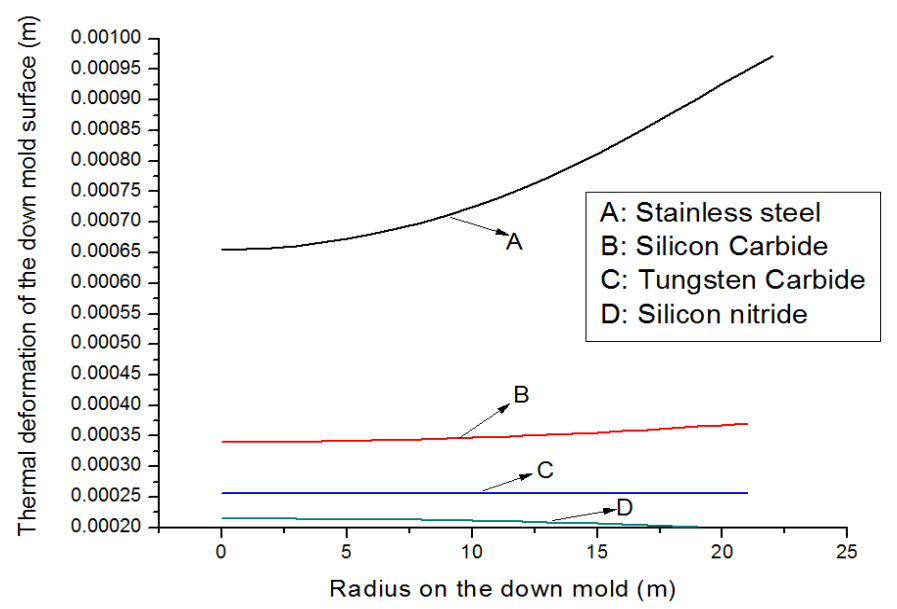

Fig. 4. Thermal deformation of the down mold surface

\section{Conclusions}

After numerical analysis combined thermal and deformation for the hot-pressing furnace, several conclusions are obtained:

1) NIR emitter is the best heat source for its high efficiency and absorption coefficient in metal.

2) One stainless steel shielding layer combining with flexible silica aerogel thermal insulation material is the more effective thermal protection measure.

3) With the wall thickness of pressing rod increasing, temperature of the glass sample decreased significantly.

4) $\mathrm{Si}_{3} \mathrm{~N}_{4}$ is a relatively better material for the pressing rods.

\section{Acknowledgements}

This research is supported by the National Science Foundation of China (No.51075381) and 2011 Young Teachers Innovation Fund of University of Science and Technology of China.

\section{References}

[1] Yi, A.Y. and A. Jain, Journal of the American Ceramic Society, 2005. 88(3): p. 579-586.

[2] W. Iqbal, Identifying the Optimum process parameters of precision glass molding for aspherical lens, 2009.

[3] F. M. Schmidt, Y. L. Maoult, and S. Monteix, J. Materials Processing Technology, vol. 143-144, 2003, p. 225-231.

[4] Tao B, He P, Shen L, Yi A. ASME. J. Manuf. Sci. Eng. 2013; 136(1):011008-011008-8.

[5] Shi, T.Y., Shen, L.G., et al., in Manufacturing Process and Equipment, Pts 1-4, X.H. Liu, K.F. Zhang, and M.Z. Li, Editors. 2013, Trans Tech Publications Ltd: Stafa-Zurich. p. 724-727.

[6] A. S. Dorcheh and M. H. Abbasi, J. Materials Processing Technology, vol. 199, 2008, pp. 10-26.

[7] F. L. Riley, J. Am. Ceram. Soc. vol. 83, pp. 245-265, 2000.

[8] M. K. Budinski, J. C. Pulver, J. J. Nelson, E. G. Hill, and D. A. Richards, Glass Mold Material for Precision Glass Molding, U.S. Patent 6363 747, 2002. 